\title{
ABORDAGEM INTERDISCIPLINAR EM MOLARES DE PACIENTES JOVENS, O DESAFIO PARA EVITAR A EXTRAÇÃO PREMATURA: RELATO DE CASOS
}

\author{
INTERDISCIPLINARY PROCEDURES ON MOLARS OF YOUNG PATIENTS, THE \\ CHALLENGE TO AVOID PREMATURE EXTRACTION: CASE REPORTS
}

\author{
Thaís Christina CUNHA'; Carlos José SOARES²; Priscilla Barbosa Ferreira SOARES; Marco Aurélio Gagliardi BORGES4; \\ Camilla Christian Gomes MOURA \\ 1 - Doutoranda em Odontologia, Faculdade de Odontologia, Universidade Federal de Uberlândia, Uberlândia - MG, Brasil. \\ 2 - Professor Adjunto 4 do Departamento de Odontologia Restauradora, Faculdade de Odontologia, Universidade Federal de Uberlândia, \\ Uberlândia - MG, Brasil. \\ 3 - Professora do Departamento de Periodontia, Faculdade de Odontologia, Universidade Federal de Uberlândia, Uberlândia - MG, Brasil. \\ 4 - Professor de Endodontia, Associação Odontológica de Ribeirão Preto, Ribeirão Preto - SP, Brasil. \\ 5 - Professora do Departamento de Endodontia, Faculdade de Odontologia, Universidade Federal de Uberlândia, Uberlândia - MG, Brasil.
}

\section{RESUMO}

O tratamento reabilitador de molares jovens severamente destruídos por cárie pode ser extremamente desafiador mesmo para profissionais mais experientes, necessitando de atendimento integrado que envolva intervenção endodôntica, periodontal e restauradora. Este artigo apresenta relato de dois casos clínicos que envolveram condução clínica bem sucedida em molares de adolescentes, severamente destruídos por cárie com lesão periapical, enfatizando protocolos interdisciplinares com acompanhamento de 1 ano. Dois pacientes do sexo masculino, 14 e 17 anos, foram atendidos no projeto de extensão de intervenção integrada na hebiatria sendo submetidos ao tratamento endodôntico dos dentes 46 e 36. Após preparo biomecânico realizado com sistema automatizado TF Adaptive (SybronEndo, Orange, CA, EUA), a limpeza e desinfecção foi completada com limas XP Endo Finisher (FKG Dentaire SA, Suíça) e irrigação final com pontas de ultrassom. Após 14 dias de curativo de demora com hidróxido de cálcio, os canais foram obturados e os dentes restaurados com restauração direta em resina composta bulk-fill (Opus Bulk Fill APS, FGM). Reparo ósseo periapical e ausência de sinais e sintomas foram observados após 6 meses e 1 ano. O protocolo apresentado favoreceu a manutenção de molares jovens após um ano de proservação.

PALAVRAS-CHAVE: Endodontia; Preparo do canal radicular; Resina composta; Resultados clínicos.

\section{INTRODUÇÃO}

A despeito da considerável redução dos índices de cárie observados em vários países ${ }^{1-3}$, essa é ainda uma realidade que acomete de forma marcante a população de baixa renda, sobretudo em países subdesenvolvidos ${ }^{4-6}$. Se não tratada precocemente, a cárie dental poderá gerar grande comprometimento estrutural, atingindo a polpa e consequentemente os tecidos perirradiculares ${ }^{7}$. Devido a fatores socioeconômicos e culturais, os responsáveis, muitas vezes, negligenciam o tratamento de cáries em crianças e adolescentes, levando a perda prematura dos primeiros molares, e consequentemente a problemas oclusais, de desenvolvimento do aparelho estomatognático e de eficiência mastigatória ${ }^{8}$.

O fato de a família geralmente procurar tratamento apenas quando o dente apresenta comprometimento pulpar, resultando em dor espontânea e elevada destruição torna necessário à realização de tratamento endodôntico radical $^{7}$. A reabilitação da função mastigatória desses dentes com pouca estrutura coronária dental remanescente representa desafio operacional com prognóstico incerto ${ }^{7}$. As dificuldades técnicas para realização do tratamento endodôntico, na fase hebiátrica, vão desde a complexidade na instalação do isolamento absoluto, instrumentação e obturação de canais geralmente amplos, além da baixa colaboração do paciente 9 .

Atualmente, o uso de instrumentos rotatórios de níquel titânio domina a prática endodôntica. Porém, estes instrumentos não foram desenvolvidos para canais amplos, pois apresentam diâmetro apical inferior a $0.5 \mathrm{~mm}$, limitando o preparo em molares jovens. Dessa forma, a lima apical final de diferentes sistemas não trabalha adequadamente as paredes dentinárias na região próxima ao ápice em dentes jovens, necessitando complementação do preparo biomecânico. Recentemente, uma nova lima, XP-Endo Finisher (\# 25 /.00, XPF, FKG Dentaire, La Chaux de Fonds, Suíça), foi introduzida como instrumento universal complementar ao preparo radicular, completando a limpeza de canais com morfologias complexas em regiões de difícil acesso ${ }^{10,11}$.

Dessa forma, o objetivo desse artigo foi apresentar dois casos de gerenciamento de protocolo integrado bem-sucedido de 
dentes molares permanentes de pacientes jovens, severamente destruídos por cárie com lesão periapical em que se utilizou a lima XP Endo Finisher como recurso auxiliar, seguida da restauração direta com resina composta bulk fill para reabilitação da função mastigatória com recuperação biomecânica satisfatória imediata.

\section{RELATO DE CASOS}

Este relato de caso foi construído de acordo com as diretrizes da CARE 201316. Os pais dos pacientes assinaram termo de consentimento livre e esclarecido, autorizando a realização do tratamento e a publicação do caso e de quaisquer imagens que os acompanhem. Dois pacientes do sexo masculino de 14 e 17 anos foram submetidos a tratamento endodôntico nos molares inferiores direito (dentes 46, caso 1) e esquerdo (dentes 36, caso 2). A análise detalhada do histórico médico e odontológico dos pacientes não revelou doença sistêmica e condições bucais contributivas. Os exames intraorais mostraram presença de cárie com extensa perda de estrutura dentária, e ausência completa de pelo menos duas paredes coronárias (Figura 1A). Os testes diagnósticos revelaram respostas negativas aos testes de sensibilidade pulpar e palpação na região apical, ausência de mobilidade patológica, mas ambos relataram respostas ligeiramente
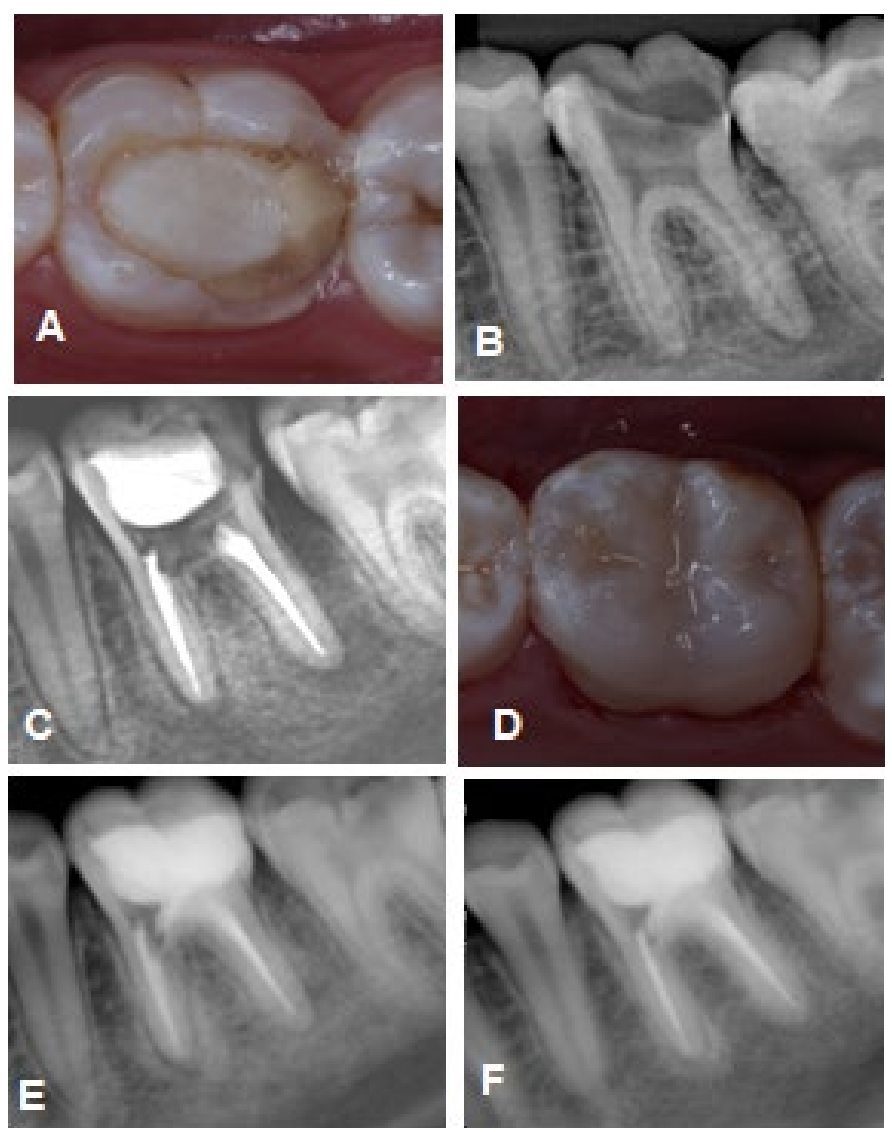

Figura 1 - Caso clínico 1; A. Imagem inicial do dente com cárie extensa resultando em comprometimento estrutural; B. Radiografia Inicial evidenciando área radiolúcida periapical na raiz distal; $C$. Radiografia final evidenciando área radiolúcida apical nas duas raízes e reduzida espessura dentinária no assoalho da cavidade; D. Dente reconstruído com resina composta bulk fill após tratamento endodôntico; E. Radiografia de controle após 6 meses, evidenciando reparo completo da lesão na raiz distal; F. Radiografia de controle após 1 ano mostrando dente completamente reparado. exacerbadas à percussão. $\mathrm{O}$ exame radiográfico revelou lesões radiolucentes periapicais (Figuras 2A e 1B), estado pulpar e perirradicular com necrose pulpar e periodontite apical assintomática, respectivamente.

No caso 2 (dente 46), após avaliação integrada das áreas de periodontia, endodontia e restauradora, foi verificada necessidade de restabelecimento do espaço biológico e reconstrução coronária para permitir isolamento absoluto adequado. Dessa forma, primeiramente foi realizada remoção do tecido cariado com instrumento manual e broca carbide esférica $n^{\circ} 3$ de baixa rotação (KG Sorensen, Barueri, SP, Brasil) de haste longa, compatível com a anatomia da câmara pulpar. Após refinamento da abertura coronária a neutralização dos condutos foi realizada com $\mathrm{NaOCl}$ 2,5\% (Rioquímica Ltda., São José do Rio Preto, SP, Brasil) sob isolamento relativo. A entrada dos condutos foi preenchida com pasta de hidróxido de cálcio (UltraCalTM XS, Ultradent Products Inc., South Jordan, UT, EUA), inserção de bolinha de algodão estéril, e selamento provisório com ionômero químico Vidrion R (SS White, Rio de Janeiro, Brasil). Tal procedimento é recomendado previamente à realização de cirurgias periodontais e restaurações transcirúrgicas a fim de se evitar a agudização do quadro clínico após a intervenção. Devido à extensa perda de estrutura dentinária na região lingual com invasão das distâncias biológicas, foi realizada cirurgia à retalho para aumento de coroa clínica e reconstrução coronária imediata com resina composta em restauração transcirúrgica (Figura 2B). Foi realizado condicionamento ácido seletivo com ácido fosfórico a $37 \%$
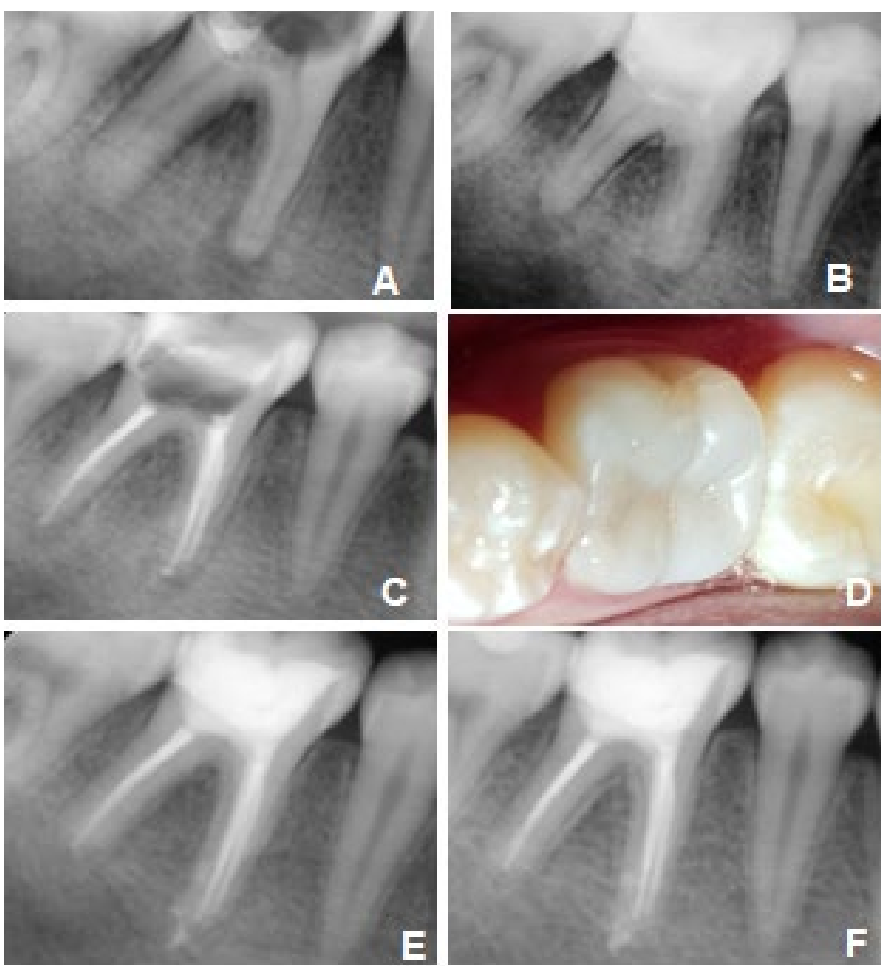

Figura 2 - Caso Clínico 2: A. Radiografia Inicial evidenciando área radiolúcida na região de furca e periápice da raiz mesial; B. Radiografia após realização de restauração transcirúrgica e reconstrução do dente em resina composta bulk fill; C. Radiografia final demonstrando ligeira extrusão de cimento obturador; D. Dente reconstruído após tratamento endodôntico; E. Radiografia de controle após 6 meses mostrando completo reparo em ambas as raízes; F. Radiografia de controle após 1 ano, material extravasado permanece próximo ao periápice com manutenção do reparo apical e na região de furca. 
(Condac 37, FGM, Joinville, SC, Brasil) por 30 segundos, seguido da lavagem com jato de ar água e secagem com papel absorvente. O sistema adesivo autocondicionante de frasco único (Ambar Universal APS, FGM) foi aplicado em duas camadas, seguido de leve jato de ar e fotoativação por 20 segundos com fonte de luz multi espectro VALO GRAND (Ultradent, South Jordan, UT, EUA) que apresenta irradiância de $1200 \mathrm{~mW} / \mathrm{cm} 2$, checada com sistema MARC Resin Calibrator (BlueLight, Halifax, Canada). A resina bulk fill (Opus Bulk Fill APS, FGM) foi inserida em incremento único e fotoativada com 40 segundos. Em sessão clínica posterior, houve continuidade do tratamento endodôntico.

Os tratamentos dos canais radiculares dos dois casos foram executados por um único e experiente operador, utilizando anestesia local com lidocaína a 2\% com epinefrina 1: 100.000 (Alphacaine; DFL Indústria e Comércio Ltda, Rio de Janeiro, Brasil) e isolamento absoluto. Primeiramente, foi realizado "Glide path" com limas manuais tipo Kerr 10 e 15 (Dentsply Maillefer, Ballaigues, Suíça) e $\mathrm{NaOCl}$ a 2,5\% (Rioquímica Ltda., São José do Rio Preto, SP, Brasil). Os comprimentos de trabalho foram determinados com o localizador foraminal RomiApex A-15 (Romidan Ltd., Kyriat Ono, Israel) e lima K no 15 em todos os canais radiculares. A instrumentação foi realizada a $1 \mathrm{~mm}$ do comprimento estimado do canal radicular, usando o sistema Twisted File Adaptive (TF) até a lima SM3 (\#35/.04, SybronEndo, Orange, CA, EUA) acoplada ao motor Elements (SybronEndo) em movimento adaptativo, de acordo com o protocolo do fabricante, sob irrigação abundante com $\mathrm{NaOCl}$ a 2,5\% (Rioquímica Ltda., São José do Rio Preto, SP, Brasil). Finalizada a instrumentação, a lima XP Endo Finisher (FKG Dentaire SA, Suíça) foi utilizada por 1 minuto, em contínuo movimento de rotação de acordo com as recomendações do fabricante (900 rpm e torque de $1 \mathrm{Ncm}$ ), sob irrigação abundante com $\mathrm{NaOCl}$ a 2,5\%.

A irrigação final de todos os canais foi realizada com $3 \mathrm{~mL}$ de ácido etilenodiaminotetracético (EDTA a 17\%, Biodinâmica, Ibiporã, PR, Brasil) por 3 minutos, seguido por $6 \mathrm{~mL}$ de $\mathrm{NaOCl}$ a 2,5\% agitados com ponta E1-Irrisonic (Helse Dental Technology, São Paulo, SP, Brasil) em potência mínima, acoplada ao aparelho ultrassom (EMS Piezon Master 200 Ultrassom, EMS, São Bernardo do Campo, SP, Brasil). Posteriormente, realizou-se irrigação com $5 \mathrm{~mL}$ de solução de cloreto de sódio a 0,9\% (Fresenius Kabi Brasil Ltda, Barueri, SP, Brasil), e secagem dos condutos com pontas de papel absorventes (Dentsply Maillefer, Ballaigues, Suíça) esterilizadas.

A medicação intracanal, pasta pré-fabricada a base de hidróxido de cálcio (UltraCalTM XS, Ultradent Prod, Inc., South South Jordan, UT, EUA), foi inserida em todo comprimento de trabalho permanecendo por 14 dias. Na sessão seguinte, como os dentes estavam assintomáticos, a medicação foi removida por meio de irrigação abundante associada a limas manuais. As raízes foram obturadas por meio da técnica de condensação lateral, utilizando cones de guta-percha (\#35/.04, Dentsply Maillefer, Ballaigues, Suíça) e cimento obturador Sealapex (SybronEndo) (Figuras 1C e 2C). Os acessos dos canais radiculares na câmera pulpar foram selados com ionômero de vidro modificado por resina (Riva SDI, São Paulo, SP, Brasil) e então reabilitados com resina composta bulk fill (Opus Bulk Fill APS, FGM) utilizando protocolo descrito anteriormente e limitando os incrementos a $5.0 \mathrm{~mm}$ de espessura (Figuras 1D e 2D). Os pacientes não apresentaram sintomas dolorosos e não foi necessário o uso de analgésicos durante e após o tratamento. As sessões de acompanhamento clínico e radiográfico foram realizadas aos 6 meses (Figuras 1E e 2E) e 1 ano após o tratamento (Figuras $1 \mathrm{~F}$ e 2F). Não foram relatados pelos pacientes sinais e sintomas relacionados aos respectivos dentes. Imagens radiográficas mostraram neoformação óssea na área perirradicular sugestiva de sucesso do tratamento endodôntico.

\section{DISCUSSÃO}

Os relatos de casos ainda apresentam grande relevância na literatura endodôntica, especialmente quando envolvem problemas clínicos com impacto social significante. Por meio desse tipo de relato, há a possibilidade de translação de evidências científicas que suportam tomadas de decisão de clínicos. $\mathrm{O}$ tratamento endodôntico-reabilitador de molares jovens, em faixa etária não mais considerada pediátrica e nem incorporada à clínica integrada de adultos é frequentemente negligenciada e pouco discutido na literatura, seja por meio de estudos laboratoriais ou clínicos ${ }^{7}$.

Reabilitar dentes de pacientes jovens com lesões periapicais e grande perda de estrutura dentária, com prognóstico incerto, se caracteriza como grande desafio ao cirurgião-dentista. Além das dificuldades técnicas relacionadas ao tratamento endodôntico de molares jovens, as quais são pouco discutidas na literatura $^{8}$, tem-se também o desafio restaurador, que deve sempre buscar simplificação técnica e baixo custo ${ }^{12}$. No caso clínico 2 , foi também necessária à realização de cirurgia periodontal a fim de restabelecer o espaço biológico, permitir reconstrução coronária e realização de adequado isolamento absoluto. A realização de restaurações transcirúrgicas por vezes é necessária nesses pacientes hebiátricos, como verifica-se na prática clínica do programa de extensão em Hebiatria da Faculdade de Odontologia da Universidade Federal de Uberlândia (FOUFU), fazendo com que o atendimento tenha que ser realizado de forma integrada e multidisciplinar.

Na prática endodôntica, com o uso cada vez mais frequente de sistemas automatizados, os quais não foram desenvolvidos com a especificidade para tratamentos de canais amplos, a instrumentação rotatória em molares jovens pode ser ineficiente, pois a lima pode não atuar adequadamente no terço apical. Considerando que a seleção das limas deve ser baseada na escolha da lima apical inicial, com posterior ampliação de pelo menos três instrumentos de numeração acima dessa ${ }^{13,14}$. Verificamos na rotina de nosso programa de extensão em Hebiatria que a execução de tal conceito é por muitas vezes impraticável em molares jovens. Nos dois casos apresentados, a última lima do sistema TF adaptive com diâmetro apical $0.35 \mathrm{~mm}$ não trabalhou de forma suficiente no terço apical. Assim, empregamos como alternativa para limpeza dos canais as limas de acabamento anatômico (XP-Endo Finisher e XP-Clean). Estas foram desenvolvidas para serem utilizados na limpeza final dos canais radiculares após qualquer técnica de instrumentação, a fim de remover detritos e microrganismos em áreas inacessíveis aos instrumentos convencionais ${ }^{11}$. Especificamente, nos casos relatados, a lima XP-Endo Finisher foi escolhida como estratégia para finalizar o preparo do sistema de canais radiculares, objetivando o acesso e limpeza das regiões não tocadas e que poderiam estar associadas à presença e/ou manutenção das lesões periapicais. 
XP-Endo Finisher (\# 25/.00) são instrumentos fabricados com a liga NiTi (MaxWire $\left.{ }^{\circledR}\right)$ e que, segundo o fabricante, é um material altamente flexível e que reage a diferentes níveis de temperatura. Essas limas aumentam o poder de limpeza da solução de irrigação por agitação mecânica, por meio de ação de "chicotear", eliminando detritos e microrganismos remanescentes devido ao contato mecânico com as paredes internas dos canais ${ }^{15-17}$. Além disso, elas também possuem a capacidade de remover medicação intracanal ${ }^{18-20}$, biofilme ${ }^{21}$, bem como evitar extrusão apical de $\mathrm{NaOCl}^{22}$. Seu uso nos casos clínicos descritos parece ter impactado positivamente no resultado do tratamento endodôntico, uma vez que em ambos os casos, as lesões regrediram completamente. Outro fator de relevância atualmente é a agitação de soluções irrigadoras por meio da oscilação livre de pontas ultrassônicas com frequência de $25-30 \mathrm{kHz}$, capaz de gerar cavitação e fluxo acústico que contribuem para a eficiência da limpeza durante a irrigação do canal radicular ${ }^{23,24}$.

Em relação à medicação intracanal, optou-se pelo $\mathrm{Ca}(\mathrm{OH})_{2,} \mathrm{O}$ qual ainda é considerado padrão ouro pela literatura em casos de dentes com lesão periapical associada ${ }^{25,26}$. É importante notar na imagem radiográfica do caso 1 , que após toda remoção do tecido cariado, a espessura dentinária no assoalho ficou bastante reduzida e com sugestão do comprometimento da furca. Porém, aos 6 meses de acompanhamento, a imagem já aponta o completo reparo nessa região, demonstrando que o tratamento empregado foi extremamente efetivo e confirmando que a associação das tecnologias atualmente disponíveis na endodontia promove descontaminação adequada sem necessidade de várias trocas de medicação intracanal ${ }^{27}$. Embora pesquisas clínicas indiquem ausência de diferenças significativas quanto ao índice de sucesso de dentes com lesões periapicais obturados em sessão única ou duas sessões, esse é ainda um tema controverso na endodontia ${ }^{28,29}$.

Em relação à alternativa reabilitadora, coroas totais apresentam baixa indicação nessa faixa etária, uma vez que o indivíduo ainda apresenta crescimento facial. No entanto, as resina híbridas convencionais demandam maior tempo pela inserção em vários incrementos, com isso, resinas bulk fill surgiram como alternativa para esses $\operatorname{casos}^{12,30-32}$ mostrando desempenho mecânico e estético satisfatório ${ }^{33}$, sendo executadas em uma única sessão, com menor custo que as restaurações indiretas.

\section{CONCLUSÃO}

Os dois casos apresentados mostraram que é possível tratar de forma adequada e conservadora molares de pacientes hebiátricos com extrema destruição coronária, mantendo o dente em função na cavidade oral, e que as tecnologias atualmente disponíveis na endodontia associadas a restaurações diretas bulk fill favorecem estes tratamentos.

\section{AGRADECIMENTOS}

CAPES, FAPEMIG, CNPq, casos clínicos desenvolvidos na clínica de extensão de Hebiatria da FOUFU.

\section{REFERÊNCIAS}

01. Souza Junior LAF, Cesaro BC, Oliveira PAB, Bordin R. Dental caries: a schoolchildren epidemiological profile in a seaside town, brazil. Int J of Adv Eng Research and Science. 2020; 7(4): 245-249.
02. Santos PS, Martins-Júnior PA, Paiva SM, Klein D, Torres FM, Giacomin A, et al. Prevalence of self-reported dental pain and associated factors among eight- to ten-year-old Brazilian schoolchildren. PLoS One. 2019; 14(4): 1-13.

03. Ravaghi V, Hargreaves DS, Morris AJ. Persistent Socioeconomic Inequality in Child Dental Caries in England despite Equal Attendance. JDR Clin Trans Res. 2020; 5(2): 185-194.

04. Linjawi AI. First molar health status in different craniofacial relationships. Clin Cosmet Investig Dent. 2016; 8: 89-94

05. Maran S, Shashikiran ND, Ahirwar P, Maran P, Raj Kannojiya P, Niranjan B. Prevalence of Dental Caries and Traumatic Dental Injuries among 6- to 12-year-old Children in Bhopal City, India. Int J Clin Pediatr Dent. 2017; 10(2): 172-176.

06. Aillón IEV, Tello G, Corrêa-Faria P, Abanto J, Oliveira LB, Bönecker M. Dental Pain in Preschool Children Using the Brazilian Dental Discomfort Questionnaire and its Association with Dental Caries and Socioeconomic Factors. Ped Dent. 2020; 42(1): 22-27.

07. Al-Madi EM, AlSaleh SA, Bukhary SM, Al-Ghofaily MM. Endodontic and Restorative Treatment Patterns of Pulpally Involved Immature Permanent Posterior Teeth. Int J Dent. 2018; 24: 1-5.

08. Selwitz RH, Ismail AI, Pitts NB. Dental caries. Lancet. 2007; 369(9555): 51-59.

09. Deepti A, Shifa S, Muthu MS, Rathna Prabhu V. Apical closure of immature molar roots: a rare case report. Int J Clin Pediatr Dent. 2008; 1(1): 54-57.

10. Alves FR, Marceliano-Alves MF, Sousa JC, et al. Removal of root canal fillings in curved canals using either reciprocating single- or rotary multi-instrument systems and a supplementary step with the XP-Endo Finisher. J Endod. 2016; 42(7): 1114-1119.

11. Vaz-Garcia ES, Vieira VTL, NPSF Petitet, Moreira EJL, Lopes HP, Elias CN, Silva EJNL, Antunes HS. Mechanical Properties of Anatomic Finishing Files: XPEndo Finisher and XP-Clean. Braz Dent J. 2018; 29(2): 208-213.

12. Rodrigues MP, Soares PBF, Gomes MAB, Pereira RA, Tantbirojn D, Versluis A, Soares CJ. Direct resin composite restoration of endodontically-treated permanent molars in adolescents: bite force and patient-specific finite element analysis. J Appl Oral Sci. 2020; 28: $1-11$.

13. Card SJ, Sigurdsson A, Orstavik D, Trope M. The effectiveness of increased apical enlargement in reducing intracanal bacteria. J Endod. 2002; 28(11): 779-783.

14. Aminoshariae A, Kulild JC. Master apical file size- smaller or larger: a systematic review of healing outcomes. Int Endod J. 2015; 48(7): 639-647.

15. Carvalho MC, Zuolo ML, Arruda-Vasconcelos R, Marinho ACS, Louzada LM, Francisco PA, Pecorari VGA, Gomes BPFA. Effectiveness of XP-Endo Finisher in the reduction of bacterial load in oval-shaped root canals. Braz Oral Res. 2019; 33(21): 1-8.

16. De-Deus G, Belladonna FG, Zuolo AS, Cavalcante DM, Carvalhal JCA, Simões-Carvalho M, Souza EM, Lopes RT; Silva EJNL. XP-endo Finisher $\mathrm{R}$ instrument optimizes the removal of root filling remnants in oval-shaped canals. Int Endod J. 2019; 52(6): 899-907.

17. Reis S, Cruz VM, Hungaro MAD, Bueno CES, Vivan RR, Pelegrine RA, Bruno KF, Kato AS. Volumetric Analysis of Irrigant Extrusion in Immature Teeth after Different Final Agitation Techniques. J Endod. 2020; 46(5): 682-687.

18. Keskin C, Sariyilmaz E, Sariyilmaz O. Efficacy of XP-Endo Finisher file in removing calcium hydroxide from simulated internal resorption cavity. J Endod. 2017; 43(1): 126-130. 
19. Gokturk H, Ozkocak I, Buyukgebiz F, Demir O. Effectiveness of various irrigation protocols for the removal of calcium hydroxide from artificial standardized grooves. J Appl Oral Sci. 2017; 25(3): 290-298.

20. Turkaydin D, Demir E, Basturk FB, Övecoglu HS. Efficacy of XPEndo Finisher in the removal of triple antibiotic paste from immature root canals. J Endod. 2017; 43(9): 1528-1531.

21. Bao P, Shen $Y$, Lin J, Haapasalo M. In vitro efficacy of XP-endo Finisher with 2 different protocols on biofilm removal from apical root canals. J Endod. 2017; 43(2): 321-325.

22. Azim AA, Piasecki L, Silva Neto UX, Cruz ATG, Azim KA. XP Shaper, a novel adaptive core rotary instrument: micro-computed tomographic analysis of its shaping abilities. J Endod. 2017; 43(9): 1532-1538.

23. Jiang LM, Verhaagen B, Versluis M, Van Der Sluis LW. Evaluation of a sonic device designed to activate irrigant in the root canal. J Endod. 2010; 36(1):143-146.

24. Virdee SS, Farnell DJJ, Silva MA, Camilleri J, Cooper PR, Tomson PL. The influence of irrigant activation, concentration and contact time on sodium hypochlorite penetration into root dentine: an ex vivo experiment. Int Endod J. 2020; 53(7): 986-997.

25. Martinho FC, Gomes CC, Nascimento GG, Gomes APM, Leite FRM. Clinical comparison of the effectiveness of 7- and 14-day intracanal medications in root canal disinfection and inflammatory cytokines. Clin Oral Investig. 2018; 22(1): 523-530.

26. Barbosa-Ribeiro M, Arruda-Vasconcelos R, Jesus-Soares A, Zaia AA, Ferraz CCR, Almeida JFA, Gomes BPFA. Effectiveness of calcium hydroxide-based intracanal medication on infectious/inflammatory contents in teeth with posttreatment apical periodontitis. Clin Oral Investig. 2019; 23(6): 2759-2766.
27. Dorasani G, Madhusudhana K, Chinni SK. Clinical and radiographic evaluation of single-visit and multi-visit endodontic treatment of teeth with periapical pathology: An in vivo study. J Conserv Dent. 2013; 16(6): 484-488.

28. El Mubarak AH, Abu-bakr NH, Ibrahim YE. Postoperative pain in multiple-visit and single-visit root canal treatment. J Endod. 2010; 36(1): 36-39.

29. Vera J, Siqueira Jr JF , Ricucci D, Loghin S, Fernández N, Flores B, Cruz AG. One- versus two-visit endodontic treatment of teeth with apical periodontitis: a histobacteriologic study. J Endod. 2012; 38(8): 1040-1052.

30. Oliveira LRS, Braga SSL, Bicalho AA, Ribeiro MTH, Price RB, Soares CJ. Molar cusp deformation evaluated by micro-CT and enamel crack formation to compare incremental and bulk-filling techniques. J Dent. 2018; 74: 71-78.

31. Cerda-Rizo ER, de Paula Rodrigues M, Vilela A, Braga S, Oliveira L, Garcia-Silva TC, Soares CJ. Bonding interaction and shrinkage stress of low-viscosity bulk fill resin composites with high-viscosity bulk fill or conventional resin composites. Oper Dent. 2019; 44(6): 625-636.

32. Boaro LCC, Lopes DP, Souza ASC, Nakano EL, Ayala Perez MD, Pfeifer CS, Gonçalves F. Clinical performance and chemical-physical properties of bulk fill composites resin - a systematic review and meta-analysis. Dent Mater. 2019; 35(10): 249-264.

33. Rodrigues MP, Soares PBF, Gomes MAB, Pereira RA, Tantbirojn D, Versluis A, Soares CJ. Direct resin composite restoration of endodontically-treated permanent molars in adolescents: bite force and patient-specific finite element analysis. J Appl Oral Sci. 2020; 28: $1-11$.

\section{ABSTRACT}

The rehabilitation of young molars teeth severely affected by caries is great challenging even for experienced professionals, requiring integrated endodontics, periodontal and restorative procedures. This paper presents two clinical cases of successful protocols for treating molar of adolescents, severely compromised by caries with periapical lesions, emphasizing interdisciplinary procedures with 6 months and 1 year follow-up. Two male patients, 14 and 17 years old, underwent root canal treatment of teeth right mandibular molar and left mandibular molar. After carrying out the biomechanical preparation with the automated TF Adaptive system (SybronEndo, Orange, CA, EUA), the cleaning and disinfection process was complemented with the XP Endo Finisher files (FKG Dentaire SA, Switzerland) and final irrigation protocols with the aid of ultrasound tips. After 14 days of calcium hydroxide dressing, the root canals were filled with sealer and gutta-percha, than the teeth were restored with bulk-fill composite resin (Opus Bulk Fill APS, FGM) restorations. Bone periapical neoformation and no signs and symptoms were observed after 6 months and 1 year of followup. The presented protocols resulted in properly maintenance of the structural compromised molar teeth in after one year of observation.

KEYWORDS: Endodontics; Root canal preparation; Synthetic resin; Treatment outcome.

\section{AUTOR PARA CORRESPONDÊNCIA}

Camilla Christian Gomes Moura

Universidade Federal de Uberlândia, Faculdade de

Odontologia - Av. Pará, 1720, Bloco 4L, Anexo A,

Umuarama, Uberlândia, MG, Brasil, CEP. 38405-328

E-mail: camillamoura@ufu.br

Telefone: +55 (34) 32258119 